\title{
Interpolativity and Continuity of Similarity-Based Reasoning Fuzzy Inference
}

\author{
${ }^{*}$ Sayantan Mandal ${ }^{a}$ and ${ }^{*}$ Balasubramaniam Jayaram $^{b}$ \\ ${ }^{a}$ Department of Mathematics, SRM University - AP, Andhra Pradesh, \\ Neerukonda, India, sayantan . m@srmap. edu . in \\ ${ }^{b}$ Department of Mathematics, IIT Hyderabad, \\ Sangareddy, India, jbala@math.iith.ac. in
}

\begin{abstract}
Similarity-based reasoning is one of the well-established inference mechanisms which is widespread in the literature. An inference mechanism is required to have some properties to be an acceptable inference mechanism that can be used in an application. Interpolativity and continuity are two of the major controlling properties of an inference mechanism. In this work, we represent the SBR inference mechanism as a function between two spaces of fuzzy sets following which we ascertain the conditions on different operations involved in a similarity-based reasoning inference mechanism that satisfies the above-mentioned properties.
\end{abstract}

Keywords: Similarity-based reasoning, Matching function, Modification function, Aggregation function, Fuzzy implication, tnorm, Interpolativity, Continuity.

\section{Introduction}

Inference mechanisms using fuzzy sets play a crucial role in various fields of applications e.g. fuzzy control, expert systems, decision making, etc. The applicability of an inference mechanism depends on the different features/ properties that an inference mechanism possesses. The process of an SBR inference mechanism involves steps like fixing the underlying input and output spaces, choosing the appropriate fuzzy logic connectives, etc. Suitable choices in these steps give different varieties of Similarity-Based Reasoning (SBR) inference mechanisms with different properties. Different types of SBR inference mechanisms have been proposed in the literature with different names e.g., Plausible reasoning [7], Compatibility Modifi- cation Inference (CMI) [5], Approximate Analogical Reasoning Scheme (AARS) [21], etc.

In this article, we will discuss SBR as a fuzzy function $\tilde{\psi}: \mathscr{F}(X) \rightarrow \mathscr{F}(Y)$. Then, we specifically discuss interpolativity and continuity of an SBR inference mechanism. Towards doing this we consider some matching function defined suitably in the predefined input space and discuss some of its properties which will be useful while discussing interpolativity and continuity of an SBR inference mechanism.

\section{Spaces of Fuzzy sets and Metrics on the Space of Fuzzy Sets}

A fuzzy inference mechanism is completely based on fuzzy sets, fuzzy rule base, and fuzzy logic connectives. In this section, we recall some spaces of fuzzy sets and some metrics on those spaces. Following this, we discuss some properties of fuzzy logic connectives that will be useful in this manuscript.

\subsection{Some Spaces of Fuzzy Sets}

In this work we consider $X \subseteq \mathbb{R}$ to be closed and bounded interval and hence $X$ is totally ordered, linear and compact w.r.t. the usual topology on $\mathbb{R}$. Let $\mathscr{F}(X)$ be the space of fuzzy sets on $X$. Based on different properties of fuzzy sets be it normality, convexity, continuity, etc, we specify some sub-spaces of the universal space $\mathscr{F}(X)$. Let $\mathscr{F}_{B N}(X)$ denote the space of fuzzy sets on $X \neq \emptyset$ which are normal and have bounded support. Few other sub-spaces of $\mathscr{F}_{B N}(X)$ that will be useful in this work are as follows:

- Every $A \in \mathscr{F}_{B N C}(X) \subseteq \mathscr{F}_{B N}(X)$ is convex.

- Every $A \in \mathscr{F}_{B N C C}(X) \subseteq \mathscr{F}_{B N C}(X)$ is also continuous.

- Every $A \in \mathscr{F}_{B N S}(X) \subseteq \mathscr{F}_{B N}(X)$ is also symmetric. 


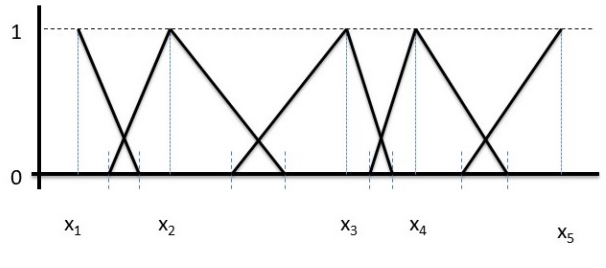

Figure 1: An Illustrative Example for $\frac{1}{3}$-type covering in Definition 2.3.

\subsection{Partitions}

Definition 2.1. Let $\mathscr{P}_{X}$ be a finite collection of fuzzy sets of $X$, i.e, $\mathscr{P}_{X}=\left\{A_{k}\right\}_{k=1}^{n} \subseteq \mathscr{F}(X) . \mathscr{P}_{X}$ is said to form a fuzzy covering on $X$ if

$$
X \subseteq \bigcup_{k=1}^{n} \operatorname{Supp} A_{k}
$$

In the literature, a covering $\mathscr{P}_{X}$ of $X$ as defined above is also called a complete partition. Note that there are several other approaches to and definitions of a fuzzy partition, see for instance, $[3,6,8]$

Definition 2.2. A fuzzy covering $\mathscr{P}_{X}=\left\{A_{k}\right\}_{k=1}^{n} \subseteq$ $\mathscr{F}(X)$ is said to form a Ruspini Partition if

$$
\sum_{k=1}^{n} A_{k}(x)=1 \text { for every } x \in X .
$$

Definition 2.3. Let $\left\{x_{k}\right\}_{k=1}^{n}$ be the nodes of a classical partition of $X$, i.e., $X=\bigcup_{k=1}^{n-2}\left[x_{k}, x_{k+1}\right) \cup\left[x_{n-1}, x_{n}\right]$. Let $\mathscr{P}_{X}=\left\{A_{k}\right\}_{k=1}^{n}$ be a fuzzy covering of the space $\mathscr{F}(X)$ in such a way that

- each $A_{k}$ is normal at $x_{k} \in X$, i.e., $A_{k}\left(x_{k}\right)=1$,

- Supp $A_{k}=\left(x_{k-1}+\frac{x_{k}-x_{k-1}}{3}, x_{k+1}-\frac{x_{k+1}-x_{k}}{3}\right)$ for $k=2, \ldots, n-1$, while Supp $A_{1}=\left[x_{1}, x_{2}-\frac{x_{2}-x_{1}}{3}\right)$ and $\operatorname{Supp} A_{n}=\left(x_{n-1}+\frac{x_{n}-x_{n-1}}{3}, x_{n}\right]$.

We call this type of covering as $\frac{1}{3}$-type covering.

For instance, see Fig. 1 for $n=5$.

\subsection{Metrics on the Space of Fuzzy Sets}

Towards discussing continuity of an SBR $\tilde{\psi}: \mathscr{F}(X) \rightarrow$ $\mathscr{F}(Y)$, there is need for metrics on $\mathscr{F}(X)$ and $\mathscr{F}(Y)$. Few examples of such metrics are the following.
Metric on $\mathscr{F}_{B N S}(X), \mathscr{F}_{B N C}(X)[20]$ :

$$
\tilde{d}(A, B)=\bigvee_{x \in X}|g(A(x))-g(B(x))|,
$$

where $g$ is the additive generator of a continuous tnorm, and $A, B \in \mathscr{F}_{B N S}(X)$ or $\mathscr{F}_{B N C}(X)$.

A Metric on $\mathscr{F}_{B N S}(X)[14]$ :

$$
\begin{array}{r}
\tilde{d}(A, B)=\max \{|\operatorname{Supp} A \backslash \operatorname{Supp}(A \cap B)|, \\
|\operatorname{Supp} B \backslash \operatorname{Supp}(A \cap B)|\} .
\end{array}
$$

where $A, B \in \mathscr{F}_{B N S}(X)$ and $|$.$| is the length of the inter-$ val.

\subsection{Fuzzy Logic Connectives}

The fuzzy logic connectives that will be extensively used in this work are t-norms, t-conorms and fuzzy implications [10,1].

Remark 2.4. From the definition of a fuzzy implication $I$, it can be easily verified that

$$
I(0, b)=1, \quad b \in[0,1] .
$$

It is also called the left- boundary condition of a fuzzy implication I.

Definition 2.5 ([1]). A fuzzy implication $I:[0,1]^{2} \rightarrow$ $[0,1]$ is said to satisfy the left neutrality property, if

$$
I(1, y)=y, y \in[0,1] .
$$

\section{Similarity-Based Reasoning (SBR)}

For two non-empty crisp sets $X, Y \subsetneq \mathbb{R}$, a Single Input Single Output (SISO) IF-THEN fuzzy rule base is of the form:

$$
\mathscr{R}\left(A_{i}, B_{i}\right): \text { IF } \tilde{x} \text { is } A_{i} \text { THEN } \tilde{y} \text { is } B_{i},
$$

where $\tilde{x}, \tilde{y}$ are the linguistic variables and $A_{i} \in$ $\mathscr{F}(X), B_{i} \in \mathscr{F}(Y), i=1,2, \ldots n . \quad A_{i}$ 's and $B_{i}$ 's are sometimes termed as antecedents and consequents, respectively in a rule base.

Given a SISO fuzzy rule base (2) and given any input " $\tilde{x}$ is $A^{\prime \prime}$ ", the main objective of an inference mechanism is to find a $B^{\prime}$ such that " $\tilde{y}$ is $B^{\prime}$ ". Many types of inference mechanisms are available in the literature [2], [12], [27]. Here we consider only the case of Similarity-based reasoning. Compatibility Modification Inference (CMI) [5], Approximate Analogical Reasoning Scheme (AARS) in [21] and Consequent Dilation Rule (CDR) in [19], Smets and Magrez [12], Chen [4], etc are some commonly known SBR inference schemes. In this section, we detail a typical inference mechanism in SBR considering a SISO fuzzy rule base (2). 
Let a given input be " $\tilde{x}$ is $A^{\prime \prime}$ ". In an SBR inference mechanism, the first step is to calculate the measure of compatibility or similarity between the antecedents $A_{i}, i=1,2, \ldots n$ and the input $A^{\prime}$ by a matching function. In the second step, the obtained similarity values are used to modify the respective consequents $B_{i}, i=$ $1,2, \ldots n$ through a modification function $J$ to obtain the modified consequents. In the final step, all the modified consequents are aggregated to obtain the final output $B^{\prime}$ in an SBR.

\subsection{Matching function $M$}

A matching function on the space of fuzzy sets $\mathscr{F}(X)$ is defined to be as a function $M: \mathscr{F}(X) \times \mathscr{F}(X) \rightarrow$ $[0,1]$. In essence, for any $A, A^{\prime} \in \mathscr{F}(X), M\left(A, A^{\prime}\right) \in$ $[0,1]$ represents the similarity value (sometimes also called compatibility value or matching value) between $A$ and $A^{\prime}$. Here we produce some examples of matching functions.

Example 3.1. Let $X$ be a non-empty set and $A, A^{\prime} \in$ $\mathscr{F}(X)$. Some examples of matching functions are

- Zadeh [28]: $M_{\mathbf{Z}}\left(A, A^{\prime}\right)=\max _{x \in X} \min \left(A(x), A^{\prime}(x)\right)$.

- Magrez - Smets [12]: Given a fuzzy negation N,

$$
M_{\mathbf{M}}\left(A, A^{\prime}\right)=\max _{x \in X} \min \left(N(A(x)), A^{\prime}(x)\right) .
$$

- Measure of Subsethood [19]: For a fuzzy implication I,

$$
M_{\mathbf{S}}\left(A, A^{\prime}\right)=\min _{x \in X} I\left(A^{\prime}(x), A(x)\right) .
$$

Remark 3.2. Formally, while $M: \mathscr{F}(X) \times \mathscr{F}(X) \rightarrow$ $[0,1]$, in practice, the antecedents of fuzzy rules come from a fuzzy covering $\mathscr{P}_{X}=\left\{A_{k}\right\}_{k=1}^{n} \subseteq \mathscr{F}(X)$ and hence one may restrict the matching function w.r.t. a covering $\mathscr{P}_{X}$ on $\mathscr{F}(X)$, which is nothing but a mapping:

$$
\left.M\right|_{\mathscr{P}_{X}}: \mathscr{P}_{X} \times \mathscr{F}(X) \rightarrow[0,1] .
$$

Example 3.3. Let $X \subsetneq \mathbb{R}$ be any bounded interval For a given fuzzy covering $\mathscr{P}=\left\{A_{k}\right\}_{k=1}^{n} \subseteq \mathscr{F}_{B N C C}(X)$. We may define a matching function as,

$$
\left.M\right|_{\mathscr{P}_{X}}\left(A_{k}, A^{\prime}\right)=\frac{\operatorname{Area}\left(A^{\prime} \cap A_{k}\right)}{\operatorname{Area}\left(A_{k}\right)}, A^{\prime} \in \mathscr{F}_{B N C C}(X) .
$$

A matching function is expected to have some properties to be suitably employed in an SBR. We provide definitions of some of such properties.

Definition 3.4. Let $\mathscr{F}(X)$ be an arbitrary collection of fuzzy sets on $X . M$ is said to be consistent with $\mathscr{F}(X)$ if for any $A \in \mathscr{F}(X)$,

$$
M(A, A)=1 .
$$

Definition 3.5. Let $\mathscr{P}_{X}=\left\{A_{k}\right\}_{k=1}^{n} \subseteq \mathscr{F}(X)$ be a given fuzzy covering of $\mathscr{F}(X)$ and $A \in \mathscr{F}(X) . M$ is said to be consistent with $\mathscr{P}_{X}$ if

$$
\sum_{k=1}^{n} M\left(A_{k}, A\right) \leq 1 .
$$

(MCP)

Example 3.6. Clearly $M$ defined in Example 3.3 satisfies $(\mathrm{MCF})$ and $(\mathrm{MCP})$ w.r.t. $\mathscr{F}_{B N C C}(X)$.

Example 3.7. The matching function $M_{\mathbf{Z}}$ in Example 3.1 satisfies $(\mathrm{MCF})$ on $\mathscr{F}_{B N}(X)$.

Example 3.8. Let $X \subsetneq \mathbb{R}$ be any bounded interval. Let the antecedent fuzzy sets $\mathscr{P}_{X}=\left\{A_{k}\right\}_{k=1}^{n} \subseteq \mathscr{F}(X)$ form a fuzzy covering of the type defined in Definition 2.3 of the space $\mathscr{F}(X)$. Now, if $x^{\prime} \in X$ is the input let $A^{\prime} \in \mathscr{F}(X)$ be the fuzzified input such that $A^{\prime}$ attains normality at $x^{\prime}$, i.e., $A^{\prime}\left(x^{\prime}\right)=1$. Then the matching function defined as $M_{\mathscr{P}_{X}}\left(A^{\prime}, A\right)=A\left(x^{\prime}\right)$ for any $A \in \mathscr{F}(X)$ has the properties (MCF) and (MCP).

\subsection{Modification Function $J$}

Let us recall the rule base (2)

$$
\mathscr{R}\left(A_{i}, B_{i}\right): \text { IF } \tilde{x} \text { is } A_{i} \text { THEN } \tilde{y} \text { is } B_{i} .
$$

Let $A^{\prime}$ be a fuzzy input and $s_{i}=M\left(A_{i}, A^{\prime}\right) \in[0,1], i=$ $1,2, \ldots, n$, are the matching values between $A_{i}$ s and $A^{\prime}$, respectively, obtained through a matching function $M$. Each $B_{i}$ is then modified to $B_{i}^{\prime}$ by a modification function $J:[0,1] \times \mathscr{F}(Y) \rightarrow \mathscr{F}(Y)$ as

$$
B_{i}^{\prime}(y)=J\left(s_{i}, B(y)\right)=J\left(M\left(A_{i}, A^{\prime}\right), B(y)\right), y \in Y .
$$

Remark 3.9. Note that even though $J:[0,1] \times$ $\mathscr{F}(Y) \rightarrow \mathscr{F}(Y)$, it is essentially a function acting on the corresponding membership values of $B_{i}$ to obtain the membership values of $B_{i}^{\prime}$ and hence it can be equivalently seen as just a binary operation on $[0,1]$, i.e., $J:[0,1] \times[0,1] \rightarrow[0,1]$.

Keeping this view, in the literature, see for instance, [5], [19], [21], fuzzy implications and t-norms have typically been used as modification functions.

\subsection{Aggregation Function $G$ and the final output of an SBR inference mechanism}

For the rule base of the type (2) and a fuzzy input $A^{\prime} \in \mathscr{F}(X)$, after obtaining the modified consequents, the final output of an SBR inference mechanism is obtained by aggregating all the modified consequents. The operator being used for the above mentioned aggregation is called an aggregation function for the SBR inference mechanism. The final output is of the form

$$
B^{\prime}(y)=G_{i=1}^{n}\left(J\left(M\left(A_{i}, A^{\prime}\right), B_{i}(y)\right)\right), y \in Y .
$$


Here $G$ is a function from $\mathscr{F}(Y) \times \mathscr{F}(Y) \times \ldots \mathscr{F}(Y)$ to $\mathscr{F}(Y)$ which essentially is an associative fuzzy logic operation from $\mathscr{F}(Y) \times \mathscr{F}(Y)$ to $\mathscr{F}(Y)$. In the literature, frequently used aggregation functions are $\mathrm{t}$ norms, t-conorms or a uninorms [10].

The overall inference mechanism can be seen as a function $\tilde{\psi}: \mathscr{F}(X) \rightarrow \mathscr{F}(Y)$, where $B^{\prime}=\tilde{\psi}\left(A^{\prime}\right)$ and

$$
\begin{aligned}
B^{\prime}(y) & =\tilde{\psi}\left(A^{\prime}\right)(y) \\
& =G_{i=1}^{n}\left(J\left(M\left(A_{i}, A^{\prime}\right), B_{i}(y)\right)\right), y \in Y .
\end{aligned}
$$

\section{Different Properties of an SBR}

The applicability of an inference mechanism depends on various properties like interpolativity, continuity, robustness, universal approximation capability, monotonicity, etc. Along the lines of these properties, various studies corresponding to different inference mechanisms have appeared in the literature e.g., $[9,11,22$, $25,23,16,24,17,15,18,26,13]$. In this section, we discuss interpolativity and continuity of an SBR inference mechanism.

\subsection{Interpolativity of an SBR}

For a given rule base, by interpolativity of an SBR inference mechanism, we mean when an input is identical to one of the antecedents in the given rule base the corresponding output also should be the corresponding consequent. If we consider the rule base (2) and the inference mechanism (4), and the input $A^{\prime}=A_{k}$, for a $k \in\{1,2 \ldots, n\}$ then the SBR inference mechanism to retain the interpolativity property, it must give as output $B^{\prime}=B_{k}$.

Theorem 4.1. Let us consider the following SBR model:

- $\mathscr{R}\left(A_{i}, B_{i}\right)$ as in (2) is a SISO rule base over given fuzzy coverings $\mathscr{P}_{X} \subset \mathscr{F}(X)$ and $\mathscr{P}_{Y} \subset \mathscr{F}(Y)$,

- $J$ is a fuzzy implication that satifies (NP),

- $G$ is a t-norm,

- $M$ satisfies $(\mathrm{MCF})$ and $(\mathrm{MCP})$ w.r.t. $\mathscr{P}_{X}$.

The fuzzy function $\tilde{\psi}$ obtained from the above model is interpolative, i.e., $\tilde{\psi}\left(A_{i}\right)=B_{i}$.

Proof. Let $A^{\prime}=A_{k}$ for some $k \in\{1,2, \ldots, n\}$. Then we have $s_{k}=M\left(A_{k}, A^{\prime}\right)=1$ and $s_{i}=0$ for all $i \neq k$, since
$M$ satisfies (MCF) and (MCP). So

$$
\begin{aligned}
\tilde{\Psi}\left(A^{\prime}\right)(y)= & B^{\prime}(y) \\
= & G_{i=1}^{n}\left(J\left(M\left(A_{i}, A^{\prime}\right), B_{i}(y)\right)\right) \\
= & G_{i=1}^{n}\left(J\left(s_{i}, B_{i}(y)\right)\right) \\
= & G\left(J\left(0, B_{1}(y)\right), J\left(0, B_{2}(y)\right),\right. \\
& \left.\ldots, J\left(1, B_{k}(y)\right), \ldots J\left(0, B_{n}(y)\right)\right) \\
= & G\left(1,1, \ldots, B_{k}(y), \ldots, 1\right) \\
& \lceil\because J(0, b)=1(\mathrm{LB}) \text { and } J(1, b)=b(\mathrm{NP}) \\
= & B_{k}(y) . \\
& \quad \because G \text { is a t-norm, } G(1, b)=b
\end{aligned}
$$

Theorem 4.2. Let us consider the following SBR model:

- $\mathscr{R}\left(A_{i}, B_{i}\right)$ as in (2) is a SISO rule base over given fuzzy coverings $\mathscr{P}_{X} \subset \mathscr{F}(X)$ and $\mathscr{P}_{Y} \subset \mathscr{F}(Y)$,

- $J$ is a t-norm,

- $G$ is a $\mathrm{t}$-conorm,

- $M$ satisfies (MCF) and (MCP) w.r.t. $\mathscr{P}_{X}$.

The fuzzy function $\tilde{\psi}$ obtained from the above model is interpolative, i.e., $\tilde{\psi}\left(A_{i}\right)=B_{i}$.

Proof. Using the properties of a t-norm $J(0, b)=$ 0 and $J(1, b)=b, b \in[0,1]$ and the properties of a tconorm $G(0, b)=b, b \in[0,1]$, the proof follows along the similar lines of the proof of Theorem 4.1.

\subsection{Continuity of an SBR}

In this section, we deal with continuity of an SBR inference mechanism. It essentially leads to discuss continuity of the function $\tilde{\psi}: \mathscr{F}(X) \rightarrow \mathscr{F}(Y)$. Since $\tilde{\psi}$ is composition of the matching function $M: \mathscr{P}_{X} \times$ $\mathscr{F}(X) \rightarrow[0,1]$, modification function $J:[0,1] \times \mathscr{P}_{Y} \rightarrow$ $\mathscr{F}(Y)$, and the aggregation function $G: \mathscr{F}(Y) \times \ldots \times$ $\mathscr{F}(Y) \rightarrow \mathscr{F}(Y)$, we discuss continuity of these functions in their respective domains.

\subsubsection{Continuity of $M: \mathscr{P}_{X} \times \mathscr{F}(X) \rightarrow[0,1]$}

Let $\tilde{d}_{X}$ be a metric defined on $\mathscr{F}(X)$ and $d_{[0,1]}$ be a metric defined on $[0,1]$.

Definition 4.3. Let $\mathscr{P}_{X}=\left\{A_{k}\right\}_{k=1}^{n} \subseteq \mathscr{F}(X)$ be a fuzzy covering. A matching function $M: \mathscr{F}(X) \times \mathscr{F}(X) \rightarrow$ $[0,1]$ is said to be continuous w.r.to $\mathscr{P}_{X}$, if for any $\varepsilon>$ 
$0, A_{k} \in \mathscr{P}_{X}$ and $A^{\prime}, A^{\prime \prime} \in \mathscr{F}(X)$ there exists $\delta>0$ such that

$$
\begin{aligned}
& \tilde{d}_{X}\left(A^{\prime}, A^{\prime \prime}\right)<\delta \\
& \quad \Longrightarrow d_{[0,1]}\left(M\left(A_{k}, A^{\prime}\right), M\left(A_{k}, A^{\prime \prime}\right)\right)<\varepsilon .
\end{aligned}
$$

Example 4.4. Let $\mathscr{P}_{X}=\left\{A_{k}\right\}_{k=1}^{n} \subseteq \mathscr{F}_{B N S}$ form a Ruspini partition and let $x_{k}$ be the points of normality of the fuzzy sets $A_{k}$. Let us consider the matching functions defined as follows, where the fuzzy set $A^{\prime}$ attains normality at the point $x^{\prime}$ :

$$
\begin{aligned}
& M\left(A_{k}, A^{\prime}\right)= \\
& \begin{cases}1-\frac{\left|\inf \left(\operatorname{Supp} A^{\prime}\right)-\inf \left(\operatorname{Supp} A_{k}\right)\right|}{\frac{1}{2} \cdot\left|\operatorname{Supp} A_{k}\right|}, & \text { if } x^{\prime} \in\left[x_{k}, x_{k+1}\right] ; \\
0, & \text { otherwise }\end{cases}
\end{aligned}
$$

and

$$
\begin{aligned}
& M\left(A_{k}, A^{\prime}\right)= \\
& \begin{cases}1-\frac{\left|\sup \left(\operatorname{Supp} A^{\prime}\right)-\sup \left(\operatorname{Supp} A_{k}\right)\right|}{\frac{1}{2} \cdot\left|\operatorname{Supp} A_{k}\right|}, & \text { if } x^{\prime} \in\left[x_{k}, x_{k+1}\right] ; \\
0, & \text { otherwise } .\end{cases}
\end{aligned}
$$

Then the above mentioned matching functions are continuous on $\mathscr{F}_{B N S}$ w.r.t. the metrics

$$
\begin{aligned}
\tilde{d}_{X}\left(A^{\prime}, A^{\prime \prime}\right)= & \max \left\{\left|\operatorname{Supp} A^{\prime} \backslash \operatorname{Supp}\left(A^{\prime} \cap A^{\prime \prime}\right)\right|,\right. \\
& \left.\left|\operatorname{Supp} A^{\prime \prime} \backslash \operatorname{Supp}\left(A^{\prime} \cap A^{\prime \prime}\right)\right|\right\}
\end{aligned}
$$

and

$d_{[0,1]}\left(M\left(A_{k}, A^{\prime}\right), M\left(A_{k}, A^{\prime \prime}\right)\right)=\left|M\left(A_{k}, A^{\prime}\right)-M\left(A_{k}, A^{\prime \prime}\right)\right|$.

To see this, let $\operatorname{Supp} A_{k}=\left[x_{k-1}, x_{k+1}\right]$ and $A_{k}\left(x_{k}\right)=$ 1 and let $A^{\prime}$ and $A^{\prime \prime}$ be two fuzzy sets for which $\inf \left(\operatorname{Supp} A^{\prime}\right)=a^{\prime}, \inf \left(\operatorname{Supp} A^{\prime \prime}\right)=a^{\prime \prime}, \sup \left(\operatorname{Supp} A^{\prime}\right)=$ $b^{\prime}$ and $\sup \left(\operatorname{Supp} A^{\prime \prime}\right)=b^{\prime \prime}$. When $x^{\prime}, x^{\prime \prime} \in\left[x_{k}, x_{k+1}\right]$, we have

$$
\begin{aligned}
& M\left(A_{k}, A^{\prime}\right)=1-\frac{\left|a^{\prime}-x_{k-1}\right|}{\left|x_{k}-x_{k-1}\right|} \text { and } \\
& M\left(A_{k}, A^{\prime \prime}\right)=1-\frac{\left|a^{\prime \prime}-x_{k-1}\right|}{\left|x_{k}-x_{k-1}\right|} .
\end{aligned}
$$

We have,

$$
\begin{aligned}
d_{[0,1]}( & \left.M\left(A_{k}, A^{\prime}\right), M\left(A_{k}, A^{\prime \prime}\right)\right) \\
& =\left|M\left(A_{k}, A^{\prime}\right)-M\left(A_{k}, A^{\prime \prime}\right)\right| \\
& =\left|\frac{\left|a^{\prime}-x_{k-1}\right|-\left|a^{\prime \prime}-x_{k-1}\right|}{x_{k}-x_{k-1}}\right| \\
& =\frac{|| a^{\prime}-x_{k-1}|-| a^{\prime \prime}-x_{k-1}||}{\left|x_{k}-x_{k-1}\right|} \\
& \leq \frac{\left|a^{\prime}-x_{k-1}-a^{\prime \prime}+x_{k-1}\right|}{\left|x_{k}-x_{k-1}\right|} \\
& =\frac{\left|a^{\prime}-a^{\prime \prime}\right|}{\left|x_{k}-x_{k-1}\right|} .
\end{aligned}
$$

We also have $\tilde{d}_{X}\left(A^{\prime}, A^{\prime \prime}\right)=\max \left\{\left|a^{\prime}-a^{\prime \prime}\right|,\left|b^{\prime}-b^{\prime \prime}\right|\right\}$.

Now for a given $\varepsilon$, if we assume $\delta=\varepsilon\left|x_{k}-x_{k-1}\right|$, then

$$
\begin{aligned}
& \tilde{d}_{X}\left(A^{\prime}, A^{\prime \prime}\right)<\delta \\
\Longrightarrow & \tilde{d}_{X}\left(A^{\prime}, A^{\prime \prime}\right)<\varepsilon\left|x_{k}-x_{k-1}\right| \\
\Longrightarrow & \max \left\{\left|a^{\prime}-a^{\prime \prime}\right|,\left|b^{\prime}-b^{\prime \prime}\right|\right\}<\varepsilon\left|x_{k}-x_{k-1}\right| \\
\Longrightarrow & \left|a^{\prime}-a^{\prime \prime}\right|<\varepsilon\left|x_{k}-x_{k-1}\right| \\
\Longrightarrow & \frac{\left|a^{\prime}-a^{\prime \prime}\right|}{\left|x_{k}-x_{k-1}\right|}<\varepsilon .
\end{aligned}
$$

Hence for a given $\varepsilon>0$ there exist a $\delta=\varepsilon\left|x_{k}-x_{k-1}\right|>$ 0 , such that

$\tilde{d}_{X}\left(A^{\prime}, A^{\prime \prime}\right)<\delta \Longrightarrow d_{[0,1]}\left(M\left(A_{k}, A^{\prime}\right), M\left(A_{k}, A^{\prime \prime}\right)\right)<\varepsilon$.

When $x^{\prime}, x^{\prime \prime} \notin\left[x_{k}, x_{k+1}\right]$, continuity of $M\left(A_{k}, A^{\prime}\right)$ follows trivially, since $M\left(A_{k}, A^{\prime}\right)=M\left(A_{k}, A^{\prime \prime}\right)=0$.

Let $x^{\prime} \in\left[x_{k}, x_{k+1}\right]$ and $x^{\prime \prime} \notin\left[x_{k}, x_{k+1}\right]$. Then since $A^{\prime}, A^{\prime \prime} \in \mathscr{F}_{B N S}$, using a case-by-case argument on the positioning of $a^{\prime \prime}$, we can show that $\left|x_{k}-a^{\prime}\right| \leq$ $\max \left\{\left|a^{\prime}-a^{\prime \prime}\right|,\left|b^{\prime}-b^{\prime \prime}\right|\right\}^{1}$.

$$
\begin{aligned}
d_{[0,1]}( & \left.M\left(A_{k}, A^{\prime}\right), M\left(A_{k}, A^{\prime \prime}\right)\right) \\
& =\left|M\left(A_{k}, A^{\prime}\right)-M\left(A_{k}, A^{\prime \prime}\right)\right| \\
& =|1-| \frac{a^{\prime}-x_{k-1}}{x_{k}-x_{k-1}}|-0| \\
& =\frac{|| x_{k}-x_{k-1}|-| a^{\prime}-x_{k-1}||}{\left|x_{k}-x_{k-1}\right|} \\
& \leq \frac{\left|x_{k}-a^{\prime}\right|}{\left|x_{k}-x_{k-1}\right|} \\
& \leq \frac{\max \left\{\left|a^{\prime}-a^{\prime \prime}\right|,\left|b^{\prime}-b^{\prime \prime}\right|\right\}}{\left|x_{k}-x_{k-1}\right|} .
\end{aligned}
$$

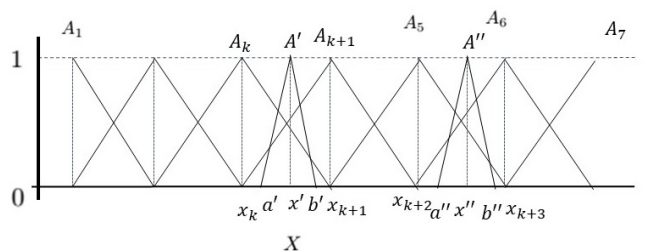

Figure 2: An Illustrative Example for the case $x^{\prime} \in$ $\left[x_{k}, x_{k+1}\right]$ and $x^{\prime \prime} \notin\left[x_{k}, x_{k+1}\right]$.

Now for a given $\varepsilon$, if we assume $\delta=\varepsilon\left|x_{k}-x_{k-1}\right|$, then

$$
\begin{aligned}
& \tilde{d}_{X}\left(A^{\prime}, A^{\prime \prime}\right)<\delta \\
\Longrightarrow & \tilde{d}_{X}\left(A^{\prime}, A^{\prime \prime}\right)<\varepsilon\left|x_{k}-x_{k-1}\right| \\
\Longrightarrow & \max \left\{\left|a^{\prime}-a^{\prime \prime}\right|,\left|b^{\prime}-b^{\prime \prime}\right|\right\}<\varepsilon\left|x_{k}-x_{k-1}\right| \\
\Longrightarrow & \frac{\max \left\{\left|a^{\prime}-a^{\prime \prime}\right|,\left|b^{\prime}-b^{\prime \prime}\right|\right\}}{\left|x_{k}-x_{k-1}\right|}<\varepsilon .
\end{aligned}
$$

${ }^{1}$ For better explanation see Figure 2 
Hence for a given $\varepsilon>0$ there exist a $\delta=\varepsilon\left|x_{k}-x_{k-1}\right|>$ 0 , such that

$\tilde{d}_{X}\left(A^{\prime}, A^{\prime \prime}\right)<\delta \Longrightarrow d_{[0,1]}\left(M\left(A_{k}, A^{\prime}\right), M\left(A_{k}, A^{\prime \prime}\right)\right)<\varepsilon$.

Remark 4.5. The matching functions in Equation (5) and (6) satisfy (MCF) and (MCP).

4.2.2 Continuity of $J:[0,1] \times \mathscr{F}(Y) \rightarrow \mathscr{F}(Y)$ and $G: \mathscr{F}(Y) \times \ldots \times \mathscr{F}(Y) \rightarrow \mathscr{F}(Y)$

Let $\tilde{d}_{Y}$ be a metric defined on $\mathscr{F}(Y)$ and $d_{[0,1]}$ be a metric defined on $[0,1]$.

Definition 4.6. The modification function $J:[0,1] \times$ $\mathscr{F}(Y) \rightarrow \mathscr{F}(Y)$ is said to be continuous at $\left(s^{\prime}, B^{\prime}\right) \in$ $[0,1] \times \mathscr{F}(Y)$, if for any $\varepsilon>0$ there exists a $\delta>0$ such that

$$
\begin{aligned}
\max \left\{d_{[0,1]}\left(s, s^{\prime}\right), \tilde{d}_{Y}\left(B, B^{\prime}\right)\right\}<\delta & \\
& \Longrightarrow \tilde{d}_{Y}\left(J(s, B), J\left(s^{\prime}, B^{\prime}\right)\right)<\varepsilon .
\end{aligned}
$$

The modification function is frequently taken as a fuzzy implication or a t-norm. It should be noted that even if the fuzzy implication or the t-norm is not continuous on $[0,1] \times[0,1]$ the modified output $J(s, B)$ may be continuous. For example, if $J(x, y)=$ $I_{\mathbf{G G}}(x, y)=\min \left\{1, y^{x}\right\}, x, y \in[0,1]$, the Yager's fuzzy implication that is not continuous and the fuzzy set $B$ is continuous, still $I_{\mathbf{G G}}(s, B)$ is continuous.

Definition 4.7. $G: \mathscr{F}(Y) \times \ldots \times \mathscr{F}(Y) \rightarrow \mathscr{F}(Y)$ is said to be continuous at $\left(B_{1}, B_{2}, \ldots B_{n}\right)$, if for any $\varepsilon>0$ there exists $\delta>0$ such that

$$
\begin{aligned}
\max _{i} & \left\{\tilde{d}_{Y}\left(B_{i}, B_{i}^{\prime}\right)\right\}<\delta \\
& \Longrightarrow \tilde{d}_{Y}\left(G\left(B_{1}, B_{2}, \ldots B_{n}\right), G\left(B_{1}^{\prime}, B_{2}^{\prime}, \ldots B_{n}^{\prime}\right)\right)<\varepsilon .
\end{aligned}
$$

Remark 4.8. The modification function $J$ should be chosen in such a way that that $J(s, B)=B^{\prime} \in \mathscr{F}(Y)$. Similar is the case for the aggregation function $G$ as well.

\subsubsection{Continuity of $\tilde{\psi}: \mathscr{F}(X) \rightarrow \mathscr{F}(Y)$}

Theorem 4.9. Let us consider the following SBR model:

- $\mathscr{R}\left(A_{i}, B_{i}\right)$ as in (2) is a SISO rule base over given fuzzy coverings $\mathscr{P}_{X} \subset \mathscr{F}(X)$ and $\mathscr{P}_{Y} \subset \mathscr{F}(Y)$,

- The modification function $J:[0,1] \times \mathscr{F}(Y) \rightarrow$ $\mathscr{F}(Y)$ is continuous.

- The aggregation function $G: \mathscr{F}(Y) \times \ldots \times$ $\mathscr{F}(Y) \rightarrow \mathscr{F}(Y)$ is continuous.

- The matching function $M$ is continuous w.r.t the fuzzy covering $\mathscr{P}_{X}=\left\{A_{k}\right\}_{k=1}^{n} \subseteq \mathscr{F}(X)$.
The function $\tilde{\psi}$ obtained from the above model is continuous.

Proof. The SBR inference mechanism $\tilde{\psi}: \mathscr{F}(X) \rightarrow$ $\mathscr{F}(Y)$ is given as

$$
B^{\prime}=\tilde{\psi}\left(A^{\prime}\right)=G_{i=1}^{n}\left(J\left(M\left(A_{i}, A^{\prime}\right), B_{i}\right)\right) .
$$

Let $B_{i}^{\prime}=J\left(M\left(A_{i}, A^{\prime}\right), B_{i}\right)$. Then we have

$$
B^{\prime}=\tilde{\psi}\left(A^{\prime}\right)=G_{i=1}^{n}\left(B_{i}^{\prime}\right)=G\left(B_{1}^{\prime}, B_{2}^{\prime}, \ldots B_{n}^{\prime}\right) .
$$

Since $G: \mathscr{F}(Y) \times \mathscr{F}(Y) \ldots \times \mathscr{F}(Y) \rightarrow \mathscr{F}(Y)$ is a continuous function, for any $\varepsilon>0$ there exists a $\delta_{2}>0$ such that

$$
\begin{aligned}
& \max _{i}\left\{\tilde{d}_{Y}\left(B_{i}^{\prime}, B_{i}^{\prime \prime}\right)\right\}<\delta_{2} \\
& \Longrightarrow \tilde{d}_{Y}\left(G\left(B_{1}^{\prime}, B_{2}^{\prime}, \ldots B_{n}^{\prime}\right), G\left(B_{1}^{\prime \prime}, B_{2}^{\prime \prime}, \ldots B_{n}^{\prime \prime}\right)\right)<\varepsilon
\end{aligned}
$$

Since $B_{i}^{\prime}=J\left(M\left(A_{i}, A^{\prime}\right), B_{i}\right)$ and $J:[0,1] \times \mathscr{F}(Y) \rightarrow$ $\mathscr{F}(Y)$ is continuous, for each $B_{i}$, corresponding to $\delta_{2}>$ 0 , there exists a $\delta_{1}^{i}>0$ such that

$$
\begin{aligned}
& \left|M\left(A_{i}, A^{\prime}\right)-M\left(A_{i}, A^{\prime \prime}\right)\right|<\delta_{1}^{i} \Longrightarrow \\
& \tilde{d}_{Y}\left(J\left(M\left(A_{i}, A^{\prime}\right), B_{i}\right), J\left(M\left(A_{i}, A^{\prime \prime}\right), B_{i}\right)\right)<\delta_{2}
\end{aligned}
$$

Letting $\delta_{1}=\min _{i} \delta_{1}^{i}$, we see that, for any $i \in$ $\{1,2, \ldots n\}$, whenever $\left|M\left(A_{i}, A^{\prime}\right)-M\left(A_{i}, A^{\prime \prime}\right)\right|<\delta_{1}$ we have $\tilde{d}_{Y}\left(J\left(M\left(A_{i}, A^{\prime}\right), B_{i}\right), J\left(M\left(A_{i}, A^{\prime \prime}\right), B_{i}\right)\right)<\delta_{2}$.

Again, since $M: \mathscr{F}(X) \times \mathscr{F}(X) \rightarrow[0,1]$ is continuous w.r.t. the fuzzy covering $\mathscr{P}_{X}=\left\{A_{k}\right\}_{k=1}^{n} \subseteq \mathscr{F}(X)$, for an arbitrary but fixed $i=1,2, \ldots n$, each $M\left(A_{i}, A^{\prime}\right)$ is continuous on $\mathscr{F}(X)$ i.e, for the given $\delta_{1}>0$ there exists a $\delta^{i}>0$ such that

$$
\tilde{d}_{X}\left(A^{\prime}, A^{\prime \prime}\right)<\delta^{i} \Longrightarrow\left|M\left(A_{i}, A^{\prime}\right)-M\left(A_{i}, A^{\prime \prime}\right)\right|<\delta_{1}
$$

Once again, letting $\delta=\min _{i} \delta^{i}$, we see that, for any $i \in\{1,2, \ldots n\}$, whenever $\tilde{d}_{X}\left(A^{\prime}, A^{\prime \prime}\right)<\delta$ we have $\left|M\left(A_{i}, A^{\prime}\right)-M\left(A_{i}, A^{\prime \prime}\right)\right|<\delta_{1}$.

The above series of arguments lead to the following: for any given $\varepsilon>0$ there exists a $\delta>0$ s.t.

$$
\begin{aligned}
\tilde{d}_{X}\left(A^{\prime}, A^{\prime \prime}\right) & <\delta \\
& \Longrightarrow \tilde{d}_{Y}\left(B^{\prime}, B^{\prime \prime}\right)<\varepsilon \\
& \Longrightarrow \tilde{d}_{Y}\left(\tilde{\psi}\left(A^{\prime}\right), \tilde{\psi}\left(A^{\prime \prime}\right)\right)<\varepsilon .
\end{aligned}
$$

Hence $\tilde{\psi}: \mathscr{F}(X) \rightarrow \mathscr{F}(Y)$ is continuous. 


\section{Concluding Remarks}

In this work, viewing an SBR inference mechanism as a function from a space of fuzzy sets to another space of fuzzy sets, we have determined the conditions on the involved functions, viz.. Matching, Modification and Aggregation functions, which are involved in an SBR inference mechanism that make it interpolative. Furthermore, we have discussed the continuity of the above-mentioned functions which finally lead to continuity of an SBR inference mechanism.

\section{References}

[1] M. Baczyński, B. Jayaram, Fuzzy Implications, Vol. 231 of Studies in Fuzziness and Soft Computing, Springer, 2008.

[2] W. Bandler, L. J. Kohout, Semantics of implication operators and fuzzy relational products, International Journal of Man-Machine Studies 12 (1) (1980) $89-116$.

[3] D. Butnariu, Additive fuzzy measures and integrals I, Journal of Mathematical Analysis and Applications 93 (2) (1983) 436 - 452.

[4] S.-M. Chen, A new approach to handling fuzzy decision-making problems, in: Multiple-Valued Logic, 1988., Proceedings of the Eighteenth International Symposium on, 1988, pp. 72-76.

[5] V. Cross, T. Sudkamp, Fuzzy implication and compatibility modification, in: IEEE International Conference on Fuzzy Systems, 1993, pp. 219-224 vol.1.

[6] B. De Baets, R. Mesiar, T-partitions, Fuzzy Sets and Systems 97 (2) (1998) 211 - 223.

[7] D. Dubois, H. Prade, The generalized modus ponens under sup-min composition: a theoretical study, in: M. Gupta, A. Kandel, W. Bandler, J. Kiszka (Eds.), Approximate Reasoning in Expert Systems, Elsevier/North-Holland, 1985, pp. 217-232.

[8] U. Höhle, Many-valued equalities, singletons and fuzzy partitions, Soft Computing 2 (3) (1998) 134-140.

[9] B. Jayaram, Rule reduction for efficient inferencing in similarity based reasoning, International Journal of Approximate Reasoning 48 (1) (2008) 156-173.

[10] E. P. Klement, R. Mesiar, E. Pap, Triangular Norms, Vol. 8 of Trends in Logic, Kluwer Academic Publishers, Dordrecht, 2000.
[11] Y.-M. Li, Z.-K. Shi, Z.-H. Li, Approximation theory of fuzzy systems based upon genuine manyvalued implications: SISO cases, Fuzzy Sets and Systems. 130 (2) (2002) 147-157.

[12] P. Magrez, P. Smets, Fuzzy modus ponens: A new model suitable for applications in knowledge-based systems, International Journal of Intelligent Systems 4 (2) (1989) 181-200.

[13] S. Mandal, Monotonicity of the system function of a SISO FRI system with neutrality and ordering property preserving fuzzy implications, International Journal of Approximate Reasoning 120 (2020) 92-101.

[14] S. Mandal, B. Jayaram, Approximation capability of SISO SBR fuzzy systems based on fuzzy implications, in: Proceedings of 6th International Summer School on Aggregation Operators, 2011, pp. 105-110.

[15] S. Mandal, B. Jayaram, Similarity-based reasoning fuzzy systems and universal approximation, in: Mathematics and Computing 2013, Vol. 91 of Springer Proceedings in Mathematics \& Statistics, Springer India, 2014, pp. 215-230.

[16] S. Mandal, B. Jayaram, Bandler-Kohout subproduct with Yager's classes of fuzzy implications, IEEE Transactions on Fuzzy Systems 22 (3) (2014) 469-482.

[17] S. Mandal, B. Jayaram, SISO fuzzy relational inference systems based on fuzzy implications are universal approximators, Fuzzy Sets and Systems 277 (2014) $1-21$.

[18] S. Mandal, B. Jayaram, Monotonicity of SISO fuzzy relational inference with an implicative rule base, IEEE Transactions on Fuzzy Systems 24 (6) (2016) 1475-1487.

[19] N. N. Morsi, A. A. Fahmy, On generalized modus ponens with multiple rules and a residuated implication, Fuzzy Sets and Systems 129 (2) (2002) $267-274$.

[20] I. Perfilieva, S. Lehmke, Correct models of fuzzy IF-THEN rules are continuous, Fuzzy Sets and Systems 157 (24) (2006) 3188-3197.

[21] I. Turksen, Z. Zhong, An approximate analogical reasoning approach based on similarity measures, IEEE Transactions on Systems, Man and Cybernetics 18 (6) (1988) 1049 -1056. 
[22] E. Van Broekhoven, B. De Baets, Monotone Mamdani-Assilian models under mean of maxima defuzzification, Fuzzy Sets and Systems 159 (21) (2008) $2819-2844$.

[23] M. Štěpnička, U. Bodenhofer, M. Daňková, V. Novák, Continuity issues of the implicational interpretation of fuzzy rules, Fuzzy Sets and Systems 161 (14) (2010) 1959 - 1972.

[24] M. Štěpnička, B. De Baets, Implication-based models of monotone fuzzy rule bases, Fuzzy Sets and Systems 232 (2013) $134-155$.

[25] M. Štěpnička, B. Jayaram, On the suitability of the Bandler-Kohout subproduct as an inference mechanism, IEEE Transactions on Fuzzy Systems 18 (2) (2010) 285-298.

[26] M. Štěpnička, S. Mandal, Fuzzy inference systems preserving Moser-Navara axioms, Fuzzy Sets and Systems 338 (2018) 97-116.

[27] L. A. Zadeh, Outline of a new approach to the analysis of complex systems and decision processes, IEEE Transactions on Systems, Man and Cybernetics SMC-3 (1) (1973) 28 -44.

[28] L. A. Zadeh, The concept of a linguistic variable and its application to approximate reasoning-III, Information Sciences 9 (1975) 43-80. 